\title{
FORMULATION AND EVALUATION OF STAVUDINE LOADED POLYMETHACRYLIC ACID NANOPARTICLES
}

\author{
Mallamma.T*. Dr. Bharathi D.R., R. Yogananda, G. Lakshmi Radhika, Vyjayanthimala T. \\ Department of Pharmaceutics, S.J.M. College of Pharmacy, N.H-4, SJMIT Campus, \\ Chitradurga, India
}

\begin{abstract}
Nanoparticles represent a promising drug delivery system of controlled and targeted drug release. They are specially designed to release the drug in the vicinity of target tissue. The aim of this study was to prepare and evaluate polymethacrylic acid nanoparticles containing stavudine in different drug to polymer ratio by nanoprecipitation method to be $121 \pm 8$ to $403 \pm 4 \mathrm{~nm}$. The particle size of the nanoparticles was gradually increased with increase in the proportion of polymethacrylic acid polymer. The drug content of the nanoparticles was increasing on increasing polymer concentration up to a particular concentration. No appreciable difference was observed in the extent of degradation of product during 60 days in which, nanoparticles were stored at various temperatures. FT-IR studies indicated that there was no chemical interaction between drug and polymer and stability of drug. The in-vitro release behavior from all the drug loaded batches was found to be zero order and provided sustained release over a period of $24 \mathrm{~h}$. The developed formulation overcome and alleviates the drawbacks and limitations of stavudine sustained release formulations and could possibility be advantageous in terms of increased bioavailability of stavudine.
\end{abstract}

Keywords: nanoparticles, stavudine, biodegradable, polymethacrylic acid.

\section{Introduction}

During last two decades, considerable attention has been given to the development of novel drug delivery system (NDDS) ${ }^{1}$. The rational for control drug delivery is to alter the pharmacokinetics and pharmacodynamics of drug substance in order to improve the therapeutic efficacy and safety through the use of novel drug delivery system. Besides more traditional matrix or reservoir drug delivery system, colloidal drug delivery system has gained in popularity. The major colloidal drug delivery system includes liposome and polymeric nanoparticles. These systems have been investigated primarily for site specific drug delivery, for controlled drug delivery, and also for the enhancement of dissolution rate/bioavailability of poorly water-soluble drugs. The primary routes of administration under investigation are parenteral route; however, other routes such as the oral, ocular, or topical routes are also being investigated.

\footnotetext{
${ }^{*}$ Correspondence to author:

Mallamma.T.

Email-mallammareddy89@gmail.com
} 
In era of oral drug delivery system microsphere ${ }^{2}$, microcapsule, nanoparticles, liposomes, and niosomes are better options to conventional dosage form. Nanoparticles are colloidal polymer particles of a size below $1 \mathrm{~mm}^{3-4}$ and hold promise as drug delivery for parenteral, peroral and ocular administration as well as adjuvant for vaccines. Due to their greater stability and due to their easier manufacturing they offer advantages over other colloidal carriers such as liposomes and cell ghosts. They offer advantages like increased bioavailability, site specific drug delivery, sustained release of drug over longer period of time, retention of dosage form in entire length of gastrointestinal tract and convenient to patient due to reduction in frequent dosing ${ }^{5}$. Eudragit polymers are series of acrylate and methacrylate polymer available in different ionic forms. Eudragit RS 100 is insoluble in aqueous media but it is permeable and has $\mathrm{pH}$ - independent release profile. The permeability of Eudragit RS 100 is due to presence of quaternary ammonium group in their structure ${ }^{6}$. Stavudine is a potent antiviral agent used in the treatment of AID. Conventional oral formulations Stavudine are administered multiple times a day (150 mg twice daily) because of its moderate half-life (5-7 hours $)^{7-8}$. Treatment of AIDS using conventional formulations of stavudine is found to have many drawbacks, such as adverse side effects resulting from accumulation of drug in multidose therapy, poor patient compliance, and high cost. The objective of the present study was to prepare nanoparticles of stavudine to overcome some of these problems.

\section{Material and method}

Stavudine was a gift sample from Cipla Pvt. Ltd. Mumbai. Eudragit RS 100 was obtained from Rohm Pharma, Germany. Dichloromethane was procured from E. Merck Ltd., Mumbai. All other chemicals used were of analytical grade.

\section{Preparation of nanoparticles}

Nanoparticles containing stavudine were prepared using nanoprecipitation method ${ }^{9-10}$. Drug was dissolved in water, and then cosolvent (acetone) was added into this solution. A cosolvent was needed in order to make the inner phase more homogeneous. Then polymer and $150 \mathrm{mg}$ of propylene glycol were dissolved in chloroform, and this solution was added to the drug solution to form dispersion. The dispersion was added to $10 \mathrm{ml}$ of aqueous ethanol solution (70\%). After 5 minutes of mixing, the organic solvents were removed by evaporation at $35^{\circ}$ under normal pressure, nanoparticles were separated by using cooling centrifuge (10000 rpm for $20 \mathrm{~min}$ ), supernatant were removed and nanoparticles washed with water and dried at room temperature in a desicator. By following the above mentioned procedure five other batches of nanoparticles ratio of 1:1, 1:2, 1:3, 1:4 and 1:5 were prepared and named $\mathrm{F}_{1}, \mathrm{~F}_{2}, \mathrm{~F}_{3}, \mathrm{~F}_{4}$ and $\mathrm{F}_{5}$ respectively.

\section{Particle size, surface morphology and zeta potential:}

The surface morphology (roundness, smoothness, and formation of aggregates) and particle size were studied by scanning electron microscopy $(\text { SEM })^{11-12}$. Zeta potential of the best formulation (F4) was determined by zeta potential probe model DT- 300.

\section{Drug content}

Drug content was determined by centrifugation method. The redispersed nanoparticles suspension was centrifuged at $15,000 \mathrm{rpm}$ for $40 \mathrm{~min}$ at $25^{\circ}$ to separate the free drug in the supernatant. Concentration of stavudine in the supernatant was determined by UV-Vis spectrophotometyrically at $271 \mathrm{~nm}$ after suitable dilution. 


\section{Fourier Transform Infra-red Spectroscopy (FT-IR) analysis}

The FT-IR spectra of pure stavudine and Eudragit RS100 nanoparticles loaded with stavudine were recorded to check drug polymer interaction and stability of drug.

\section{In vitro release studies}

In vitro release studies were carried out by using dialysis tubes with an artificial membrane. The prepared stavudine nanoparticles and $10 \mathrm{ml}$ of phosphate buffer ph 7.4 was added to the dialysis tube and subjected to dialysis by immersing the dialysis tube to the receptor compartment containing $250 \mathrm{ml}$ of phosphate buffer $\mathrm{pH}$ 6.8. The medium in the receptor was agitated continuously using a magnetic stirrer a temperature was maintained at $37 \pm 1^{\circ}$. $5 \mathrm{ml}$ of sample of receptor compartment were taken at various intervals of time over a period of $24 \mathrm{~h}$ and each time fresh buffer was replaced. The amount of drug released was determined spectrometrically at $271 \mathrm{~nm}$.

\section{Kinetic modeling}

In order to understand the kinetic and mechanism of drug release, the result of in vitro drug release study of nanoparticles were fitted with various kinetic equation like zero order $^{13}$ (cumulative \% release vs. time), first $\operatorname{order}^{14}(\log \%$ drug remaining vs time), Higuchi's model ${ }^{15}$ (cumulative \% drug release vs. square root of time). $\mathrm{r}^{2}$ and $\mathrm{k}$ values were calculated for the linear curve obtained by regression analysis of the above plots.

\section{Stability study:}

The stability study was carried using the batch F4. The stability of drug loaded nanoparticles was evaluated in terms of its drug content ${ }^{16}$. The stability of nanoparticles was evaluated in PBS (pH 6.8). Nanoparticles formulation was incubated at $5-8^{\circ}$ and $37 \pm 1^{\circ}$ for a period of 60 days. After specified time intervals, the suspension was centrifuged at $15,000 \mathrm{rpm}$ for $1 \mathrm{~h}$, supernatant was removed and nanoparticles were dissolved in dichloromethane. After adding of water and separation, the amount of drug was detected by UV-Vis spectrophotometrically method at $271 \mathrm{~nm}$.

\section{Result and Discussion}

Stavudine nanoparticles with varying proportions of stavudine and Eudragit RS 100 were prepared by nanoprecipitation method. The scanning electron microphotograph of stavudine nanoparticles is shown in fig.1. It indicated that stavudine nanoparticles have a discrete spherical structure without aggregation. The particle size of nanoparticles varied some what among the formulation due to variation in the composition of formulations. Zeta potential of best formulation was determined and it was found $+27 \mathrm{mV}$ due to quaternary ammonium group of Eudragit. Since there was a decrease of surface potential, it could be concluded that a part of drug was absorbed on the polymeric particles. The drug content was determined by centrifugation method and it was maximum in formulation FN4. The nanoparticles exhibited an increase in drug content with an increased in the polymer ratio, up to particular concentration (1:4). A decrease in drug content was observed after that point due to the saturation capacity of polymer. In FT-IR study the characteristic peak due to pure lamivudine has appeared in the spectra of nanoparticles without any markable change in the position. It indicated that there was no chemical interaction between stavudine and Eudragit RS 100. In stability study there was no remarkable change in the drug content. This indicated that formulation was stable in storage medium condition. The in vitro release profile of all formulation is shown in fig. 2 . The release of 
stavudine mainly depended upon the polymer concentration.

The burst release of stavudine from nanoparticles at initial stage resulted from the dissolution of drug crystals on the surface of nanoparticles. On increasing polymer concentration, the release rate of lamivudine from nanoparticles decreased drastically. The in vitro release data was applied to various kinetic models to predict the drug release kinetic mechanism. The release constant was calculated from the slope of appropriate plots, and the regression coefficient $\left(r^{2}\right)$ was determined. It was found that the in-vitro drug release of nanoparticles was best explained by zero order kinetics for best formulation F4 as the plots shows highest linearity. The correlation coefficient $\left(r^{2}\right)$ was found 0.99 for F4.

\section{Conclusion}

The method of preparation of nanoparticles of stavudine was found to be simple and reproducible. The slow and constant release of stavudine from nanoparticles maintain constant drug plasma concentration thereby increasing therapeutic efficacy. This study shows that polymethacrylic acid nanoparticles could be a useful carrier for stavudine. The developed formulation overcome and alleviates the drawbacks and limitations of stavudine sustained release formulations.

TABLE 1: Formulation and physicochemical characterization of Stavudine nanoparticles

\begin{tabular}{|c|c|l|c|}
\hline $\begin{array}{l}\text { Formulation } \\
\text { code }\end{array}$ & $\begin{array}{l}\text { Drug: Polymer } \\
\text { ratio }\end{array}$ & $\begin{array}{l}\text { Drug } \\
\text { Content }\end{array}$ & $\begin{array}{l}\text { Particle } \\
\text { Size }(\mathbf{n m})\end{array}$ \\
\hline FN1 & $1: 1$ & $\begin{array}{l}58.32 \pm \\
0.02\end{array}$ & $121 \pm 8$ \\
\hline FN2 & $1: 2$ & $\begin{array}{l}64.13 \pm \\
0.08\end{array}$ & $265 \pm 5$ \\
\hline FN3 & $1: 3$ & $\begin{array}{l}70.83 \pm \\
0.03\end{array}$ & $287 \pm 9$ \\
\hline FN4 & $1: 4$ & $\begin{array}{l}64.62 \pm \\
0.02\end{array}$ & $332 \pm 5$ \\
\hline FN5 & $1: 5$ & $\begin{array}{l}59.96 \pm \\
0.04\end{array}$ & $403 \pm 4$ \\
\hline
\end{tabular}

\section{* Average of three preparation \pm S.D}




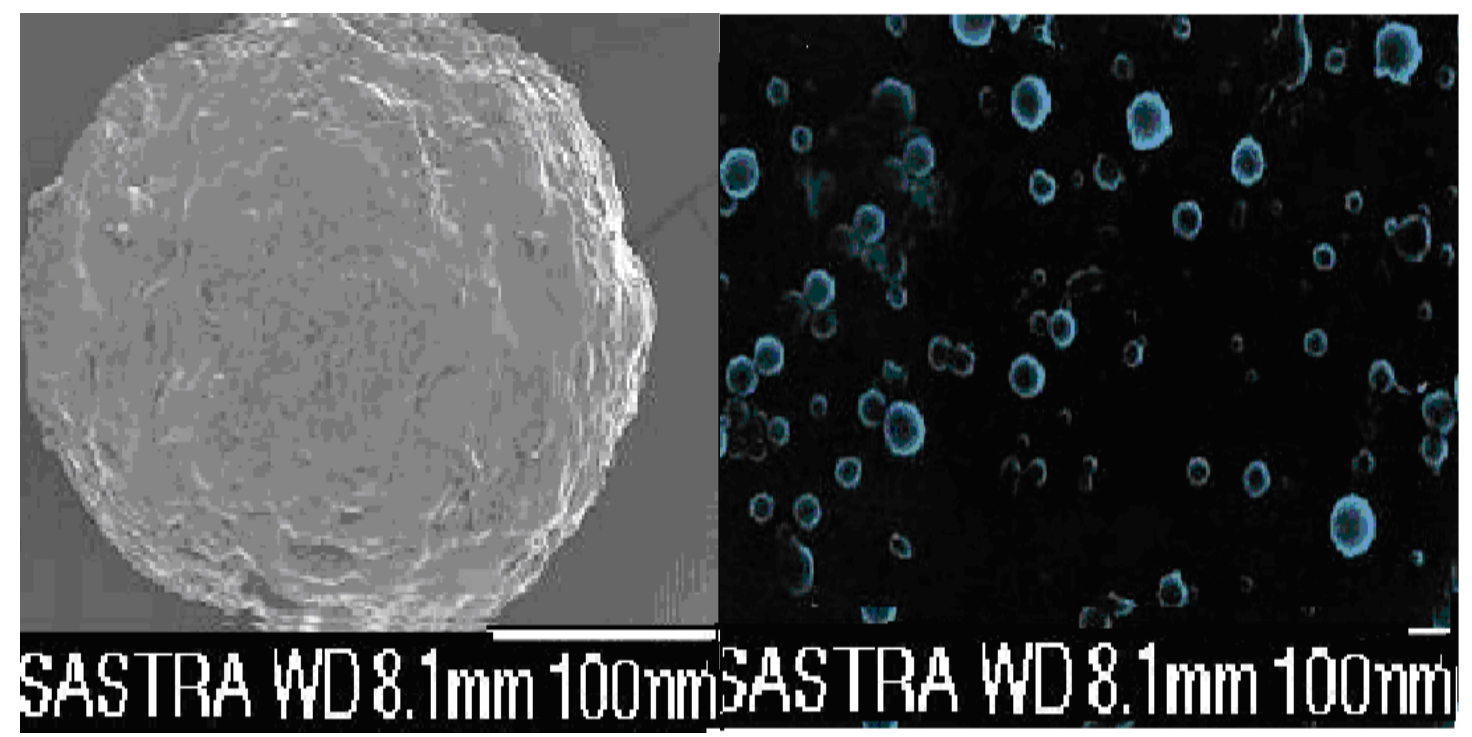

(A)

(B)

Fig.1 : Scanning electron microphotograph of stavudine hydrochloride nanoparticles (A) At lower magnification (B) At higher magnification 
Fig.2 : FT-IR spectra of pure stavudine and stavudine loaded nanoparicles formulation

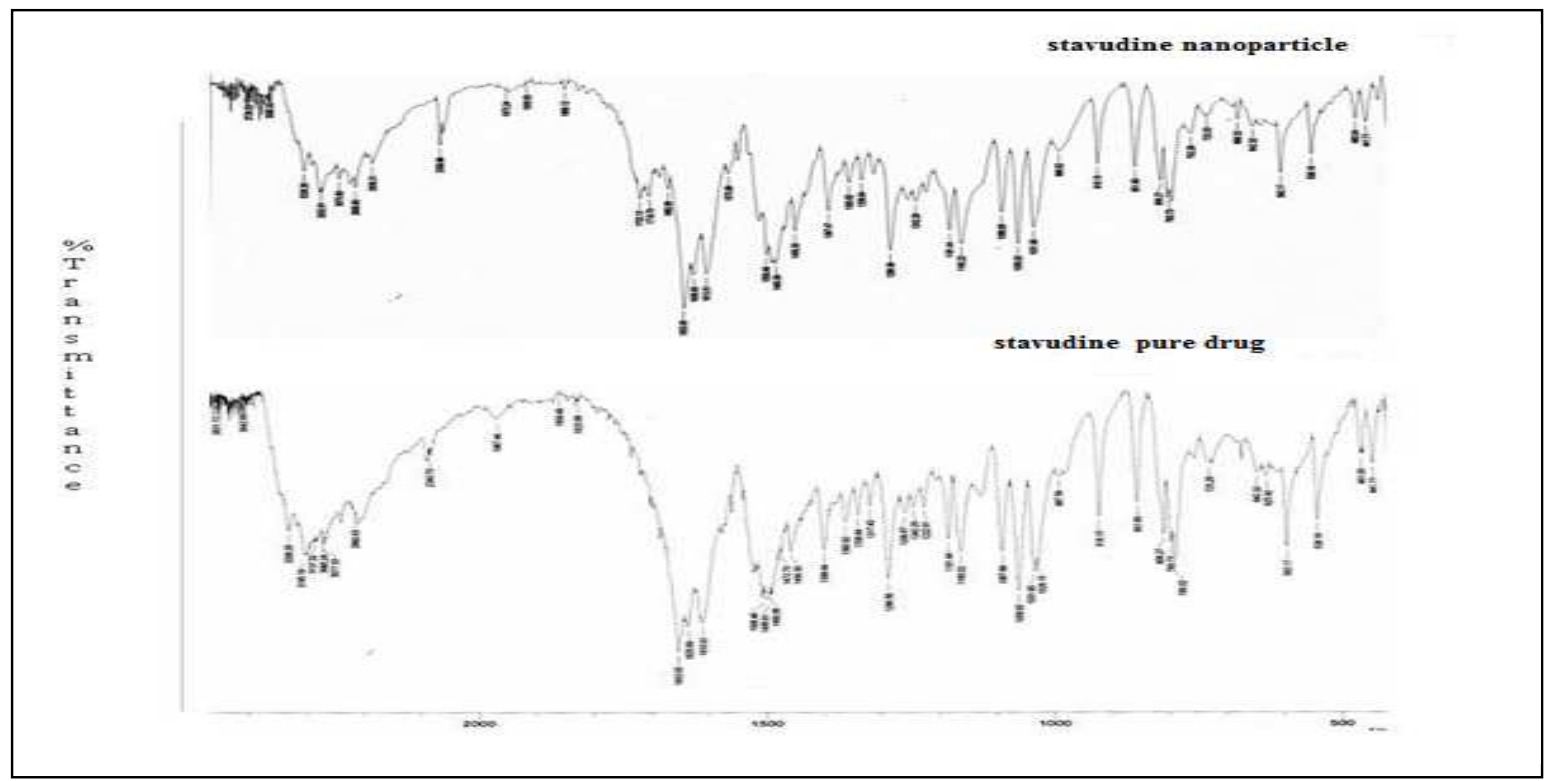

Fig.3 : In vitro drug release of stavudine from polymethaacrylic acid nanoparticles formulation FN1 (-৩-), FN2 (- -), FN3 (-D-), FN4 (-`-), FN5 (-+-).

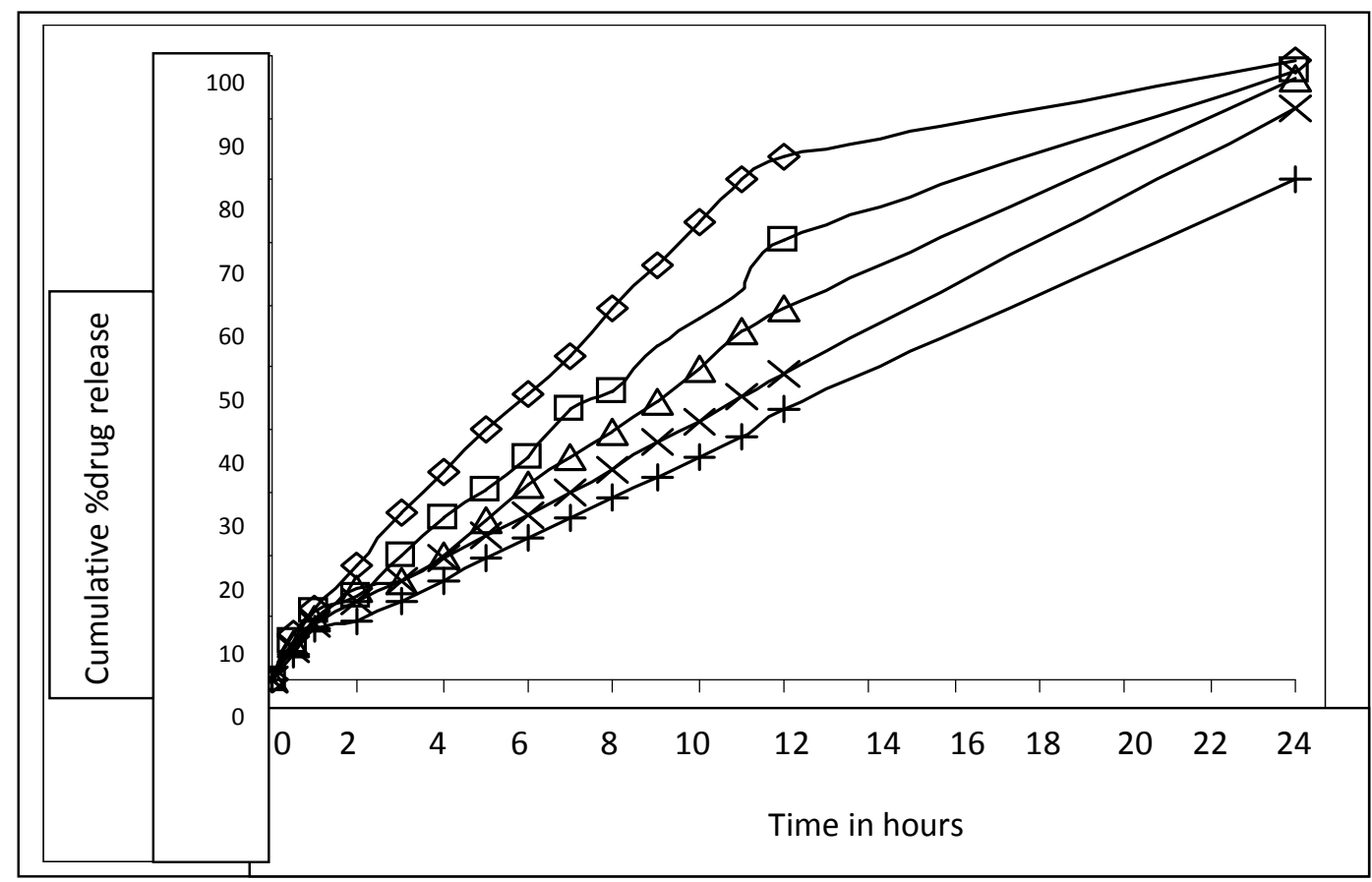




\section{References}

1. Santhi K.,Dhanraj S.A., Nagasamyvenkatesh D.,Sangeetha S.,Suresh B., Preparation and optimization of sodium alginate nanospheres of methotrexate, Indian J. Pharm. Sci., 2005, 67, 691-696.

2. Tamizharasi S.,Rathi J.C., Rathi V., Formulation, characterization and in vitro release kinetics of aceclofenac loaded poly(e-caprolactone) microspheres, Indian Drugs, 2007, 44, 973-975.

3. Kreuter J., Nanopaticles based drug delivery systems, J. Control. Rel., 1991, 16, 169-176.

4. Bachnav D., Rao M., Madgulkar A., Rao S., Nanotechnology and blood brain barrier, Indian Drug, 2007, 44, 245-252.

5. Chen D.B., Yang T. Z., Lu W. L., Zhang Q., In vitro and in vivo study of two types of long circulating solid lipid nanoparticles containing pacitaxel, Chem. Pharm. Bull., 2001, 49, 1444-1447.

6. Ubrich N., Schmidt C., Bodmeur R., Hoffman M., Maincent P., Oral evaluation in rabbits of cyclosporine loaded Eudragit RS or RL nanoparticles, Int. J. Pharm., 2005, 288,169-175.

7. Punna R.R., Sindhura G., Saha R.N., Design and Study of Lamivudine Oral Controlled Release Tablet, AAPS PharmSciTech, 2007, 8, E1-E9.

8. Jain S, Tiwari AK, Sapra B , Jain AK., Formulation \& evaluation of Ethosomes for Transdermal Delivery of Lamivudine, AAPS PharmSciTech, 2007, 8, E1-E8.

9. Leena Peltonen., The Effect of Cosolvents on the Formulation of Nanoparticles From Low- Molecular-Weight Poly(I)lactide AAPS PharmSciTech., 2002, 3, E1-E7.

10. Barbault S, Gref R., Russo P., Guechot J., Bochot A., Design of poly-ecaprolactone nanospheres coated with bioadhesive hyaluronic acid for ocular delivery, J. Control. Rel., 2002, 83, 365-375.

11. Peltonen L., Koistinen P., Karjalainen M., Hakkinen A., Hirvonen J., The effect of cosolvents on the formulation of nanoparticles from low molecular weight poly(I)lactide, AAPS PharmSciTech, 2002, 3, 1-7.

12. Cui F., Oian F., Yin C., Preparation and characterization of mucoadhesive polymer- coated nanopaticles, Int. J. Pharm., 2006, 316,154-161.

13. Saparia B., Murthy R.S.R., Solanki A., Preparation and evaluation of chloroquine phosphate microspheres using cross-linked gelatin for long term drug delivery, Indian J. Pharm. Sci., 2002, 64, 48-52. 
14. Haznedar S., Dortunc B., Preparation and evaluation of Eudragit microspheres containing acetazolamide, Int. J. Pharm.,2004, 269, 131-140.

15. Higuchi T.,Mechanism of sustained action medication: theoretical analysis of rate of release of solid drugs dispersed in solid matrices,J.Pharm. Sci., 1963, 52, 11451149.

16. Ramteke S., Maheshwari R.B.V., Jain N. K., Clarithromycin based oral sustained release nanoparticulate drug delivery system, Indian J. Pharm. Sci., 2006, 68, 479484.

17. Chowdary K.P.R., Rao Y.S., Mucoadhesive microcapsules of glipizide : Characterization, in vitro and in vivo evaluation, Indian J. Pharm. Sci., 2006, 68, 479484.

18. Chowdary K. P. R., Rao N. K., Malathi K., Ethyl cellulose microspheres of glipizide: Characterization, in vitro and in vivo evaluation, Indian J. Pharm. Sci., 2004, 66, 412-416. 